\title{
Immuno-Pathogenesis of Periodontal Disease: Current and Emerging Paradigms
}

\author{
Nasi Huang • Frank C. Gibson III
}

Published online: 22 March 2014

(C) Springer International Publishing AG 2014

\begin{abstract}
Periodontal disease (PD) is a highly complex disease involving many factors; however, two principal facets central to initiation and progression of the majority of PD are the composition of the microbes in the subgingival plaque, and the host immune response to these organisms. Numerous studies point to the complexity of $\mathrm{PD}$, and to the fact that despite innate and adaptive immune activation, and resultant inflammation, our immune response fails to cure disease. Stunning new findings have begun to clarify several complexities of the host-pathogen interaction of PD pointing to key roles for microbial dysbiosis and immune imbalance in the pathogenesis of disease. Furthermore, these investigations have identified novel translational opportunities to intercede in PD treatment. In this review we will highlight a select few recent findings in innate and adaptive immunity, and host pathogen interactions of PD at a micro-environmental level that may have profound impact on PD progression.
\end{abstract}

Keywords Periodontal disease $\cdot$ Innate immunity $\cdot$ Adaptive immunity $\cdot$ Bacteria $\cdot$ Microbial dysbiosis $\cdot$ Porphyromonas gingivalis $\cdot$ Cell receptors $\cdot$ Cytokines $\cdot$ Chemokines . Resolving · Interferon · Complement · T cell imbalance . Macrophages $\cdot$ Dendritic cells $\cdot$ Micro-environment . Osteoclast $\cdot$ Bone remodeling

\footnotetext{
N. Huang $\cdot$ F. C. Gibson III $(\bowtie)$

Section of Infectious Diseases, Department of Medicine, Boston University School of Medicine, Evans Biomedical Research Center, Room 638, 650 Albany Street, Boston, MA 02118, USA

e-mail: fgibson@bu.edu

N. Huang

e-mail: nasi@bu.edu
}

\section{Introduction}

Periodontal disease (PD) is one of the most common chronic inflammatory diseases of humans. PD is multi-factorial, with significant involvement of host, environment, and bacterial factors [1]; however, it is the host inflammatory response that drives much of the soft and hard tissue destruction. In severe disease this can lead to tooth loss. Localized forms of PD are associated with Aggregatibacter actinomycetemcomitans, while chronic generalized forms of disease are associated with a number of bacteria including Porphyromonas gingivalis, Tannerella forsythia, Treponema denticola, and others. The microbial diversity of the oral cavity is immense, and it is clear that the host response during PD is complex, with innate and adaptive elements driving chronic inflammation and bone loss. Yet, from a mechanistic level our understanding of the disease process is still emerging. Indeed, acquisition of select pathogens, with their impact on microbial communities; emergence of important new $\mathrm{T}$ cell subsets, and their implication in bone loss and preservation of oral bone; as well as the implication of the local micro-environment in PD, may have significant impact on the way the host recognizes and responds to periodontal pathogens. In PD, erosion of the bone supporting the teeth does not appear to occur in a predictable manner. Indeed, it is not clear what factors are absolutely required to define where bone loss will occur. In this review we will focus on a select few contemporary findings of the host innate and adaptive immune response present during PD, or elicited by periodontal bacteria, and discuss their impacts on inflammation and oral bone homeostasis. Generalized chronic PD represents the majority of PD, and will be the primary focus. We will illustrate novel hypotheses and findings that have recently changed our understanding of the host immune response in $\mathrm{PD}$ and its pathogenesis. 


\section{Bacterial Complexity of PD and Microbial Dysbiosis}

Early descriptions of PD note the importance of dental plaque in the progression of disease. Through detailed culture-based evaluations of dental plaque samples, it became clear that there are differences in the microbes residing in dental plaque of healthy individuals and those with PD. In PD, frequently, Gram negative organisms of the "Red complex" including $P$. gingivalis, T. forsythia, and T. denticola are detected [2]. Indeed, the seminal finding in non-human primates that $P$. gingivalis oral challenge stimulated oral bone loss [3], solidified this organism as a pathogen. Yet, insult with multiorganism complexes of periodontal pathogens can stimulate more severe forms of oral bone loss than mono-microbial challenge, thus supporting the importance of microbial complexity in disease pathogenesis $[4,5]$. Now, more powerful analysis techniques, including $16 \mathrm{~S}$ sequence analysis, provide a more thorough definition of the microbial composition of the oral cavity. Not only do these contemporary approaches better clarify the range of microbial diversity in sub-gingival plaque, but may shed light on new bacteria or groups of microorganisms associated with disease. The bacteria that inhabit the sub-gingival crevice are predominantly associated with biofilm [6]. Dewhirst et al. [7] generated sequence information and have begun to define the human oral microbiome, which consists of over 600 distinct species. Employing this tool investigators identified organisms that either increased or persisted in refractory cases of PD and identified bacteria previously not associated with disease [8•].

Recent findings suggest that periodontal pathogens can sufficiently modify their environment in a manner favorable for their survival, and stimulate microbial dysbiosis. It is suggested that this dysbiosis may be a critical element in the switch from periodontal health to disease [9]. Based on this, organisms capable of modifying their local environment such as $P$. gingivalis may serve as keystone pathogens, driving complex microbial changes that shift the host response during disease $[10,11]$. Indeed, $P$. gingivalis possesses an array of virulence factors including major and minor fimbriae, LPS, ceramides, gingipains, and others [12-15]. Gingipains are cysteine proteases, and are grouped based on enzymatic activity for arginine or lysine residues [15]. Gingipains are known to cleave a variety of host proteins including cytokines, cell adhesion molecules, complement components [15], and thus possess the ability to modify the local inflammatory environment, possibly tailoring it to one that is more favorable for $P$. gingivalis survival [16].

\section{PD Inflammatory Mediators and Immune Cell Mileau}

It is generally accepted that the host inflammatory response mounted during PD drives much of the hard and soft tissue destruction that accompanies PD. Innate and adaptive immune activation is evident in PD, with numerous immune mediators including cytokines, chemokines, acute phase mediators, and antibodies detected at levels different than in periodontal health to PD-associated organisms such as $P$. gingivalis [17-20]. Here, we will not detail the contributions of known cells to immune outcomes of PD, but will focus on a few interesting emerging themes.

\section{Inflammation}

Cellular infiltration and expression of a complex array of cytokines, chemokines, and lipid mediators are key characteristics of PD. In early / active lesions neutrophils predominate the cellular infiltrate, while in advanced chronic lesions, there is a switch to monocyte rich lesions characterized with T cells, $\mathrm{B}$ cells, dendritic cells (DC), and macrophages (MØ) [21]. These cells migrate to the nidus of infection via gradients of complement breakdown, and chemokines using an array of cell adhesion molecules.

In PD the presence of elevated inflammatory cytokines including tumor necrosis factor (TNF)- $\alpha$, interleukin (IL)-1, IL-6, interferon (IFN)- $\gamma$, and IL-12 is considered a central force, when coupled with cell activation and receptor activator of nuclear factor kappa-B ligand (RANKL) activation in driving pathogen elicited bone loss [22, 23]. In addition to pro-inflammatory cytokines, an array of chemokines including IL-8, monocyte chemotactic protein (MCP)-1, and others are frequently elevated in PD [24]. In addition, the presence of anti-inflammatory and regulatory cytokines, such as transforming growth factor (TGF)- $\beta$, IL-10, and IL- 4 , has been reported [20, 25]. In light of the complexity of this response, early investigations into the type of $\mathrm{T}$ helper (Th) cell guiding the host response to infection were undertaken, however despite an array of studies, no clear consensus has emerged (see review [26], and other reviews in this issue). Historically, differentiated T cells were divided to either Th1type, or Th2-type cells [27], and many studies have shown that both types play a role in PD progression $[28,29]$. Recently however, it has become clear that additional Th cell subsets exist, and many new studies have begun addressing the role of these T cell subsets in PD.

Among the earliest host response molecules found in response to infection are lipid mediators [30]. Resolution of inflammation involves the production of lipid mediators termed immunoresolvents, and includes the resolvins, protectins, lipoxins, and maresins (see recent reviews [21, $30,31]$ ). Resolvins are synthesized from dietary precursor essential $\omega-3$ polyunsaturated fatty acids eicosapentaenoic acid and docosahexaenoic acid [32]. Functionally, resolvins limit inflammation in part through prevention of neutrophil penetration, limiting inflammation at the local level, and promote tissue regeneration $[33,34]$. Topical treatment with 
resolvin R1 limits experimental periodontitis [35]. Based upon these important findings, targeting the reduction of inflammation through use of resolvin-based approaches may represent a novel strategy to potentially augment PD treatment approaches.

Novel T Cell Subsets and Immune Imbalance in PD

T cells are well known as key regulating cells that orchestrate the host immune response, and they can be classified based on function, including Th cells, $\mathrm{T}$ cytotoxic $(\mathrm{Tc})$ cells, and $\mathrm{T}$ regulatory (Treg) cells. Th cells arise from the $\mathrm{CD} 4^{+}$population and based on cytokine profiling early studies showed them to segregate into Th1 and Th2 populations [27].

Seymour et al. [28] suggested that the early periodontal lesion is associated with a Th1 response, while other studies support that progressive lesions possess a Th1 profile, where stable lesions possess Th2 characteristics [29]. Indeed, it is clear that there is no consensus on the contribution of $\mathrm{T}$ cell help in PD that is consistent with the Th1 versus Th2 paradigm. Modern cytokine profiling and transcription factor analysis has led to a much more detailed classification of Th cells, and the emergence of the Th17, Treg, $\mathrm{T}_{\mathrm{FH}}$, Th9, and Th22 subsets [36, 37]. Recent studies in PD report that not only do these $\mathrm{T}$ cell subsets exist, but also that a complex interaction between $\mathrm{T}$ cell subsets and an imbalance between key subsets may be crucial to PD pathogenesis [38-40, 41••]. At the heart of this imbalance lie the opposing roles of Th17 and Treg cells. Indeed, an inverse relationship between Th17 and Treg cell populations has been suggested in PD [42••]. Th17 cells associate with periodontal inflammation and tissue destruction [43], while Treg cells are implicated in protection from the development of periodontitis [40]. Employing mouse models of bone loss, it was reported that levels of both Th17 and Treg cell-related cytokines increased in response to infection, and that inhibiting IL-17 blocked periodontal destruction; whereas inhibiting the function of Treg cells exacerbated periodontal lesions $[40,41 \bullet \bullet]$.

Emerging information regarding DCs and DC / T cell interaction has shed light on aspects of the adaptive immune response during PD. Antigen presenting cells utilize MHCII coupled with co-stimulatory molecules to present antigen to $\mathrm{T}$ cells. P. gingivalis LPS can induce endotoxin tolerance [44]. DC treated with $P$. gingivalis LPS led to shifts in the expression of the T cell co-stimulatory molecules CD80 and CD86 [45]. Furthermore, chronic periodontitis may involve activation and in situ maturation of DCs in response to $P$. gingivalis with production of regulatory cytokines and the formation of T cell-DC foci [46]. In the context of poly-microbial challenge of mature DCs $P$. gingivalis / F. nucleatum and $P$. intermedia / $F$. nucleatum challenge resulted in synergized IL- 6 and TNF- $\alpha$ expression. P. intermedia / F. nucleatum combination synergized IL-12 production. These results indicate that poly- bacterial challenge of cells results in varied response profiles associated with antigen presentation and immune activation that is dependent on the specific characteristics of the microbiota present in the sub-gingival plaque [47].

With these polar groups of $\mathrm{T}$ cells present in gingival tissues, it is highly likely that the fine balance between these subsets at the micro-environmental level may be a key factor in the host transition from periodontal health to disease. Modulation of the $\mathrm{T}$ cell imbalance may provide important new clinical approaches to PD therapy (see therapy section). Furthermore, as has been reported with polymicrobial challenge, the sub-gingival microbiota likely serves as an important driver of the local inflammatory lesion, possibly by influencing shifts in $\mathrm{T}$ cells, $\mathrm{T}$ cell subsets, and other inflammatory cells present at these sites (Fig. 1).

\section{Innate Sensing of Periodontal Pathogens}

Innate recognition of bacteria and their products by the host involves a sophisticated array of receptors providing specificity to pathogen detection. Through these receptors cells can directly respond to conserved pathogen-associated microbial patterns (PAMPS), and host danger-associated molecular patterns (DAMPS).

\section{Toll-Like Receptors (TLRs) and TLR Signaling}

To understand PD, it is critical to focus on the role played by TLRs and other pathogen recognition receptors in tuning the host immune response. In the context of PD, TLR2 and TLR4 play important roles in bacterial antigen sensing [48, 49], and oral bone loss [50-52]. TLR engagement initiates intracellular signaling via adaptor molecules culminating in nuclear factor kappa-light-chain-enhancer of activated B cells (NFKB) activation and gene expression [53]. TLR signaling occurs in a manner that is dependent on the adaptor molecule myeloid differentiation primary response gene (MyD88), or occurs independent of MyD88 via TIR-domain-containing adapterinducing interferon- $\beta$ (TRIF) $[53,54]$. All TLRs with the exception of TLR3 signal via MyD88; however, TLR4 engages both MyD88 and TRIF signaling pathways. To date, TLR2 has been shown to be an important sensor of $P$. gingivalis and other periodontal pathogens. As such, MyD88 is expected to play a key role in tailoring the host innate response to periodontal pathogens. However, emerging data suggests that MyD88-independent pathways (TRIFdependent and others) may contribute significantly to PD. A seminal study from Burns et al. [55] demonstrated that although the host cytokine response to $P$. gingivalis challenge is dependent on TLR2, MyD88 is not critical. In contrast, the ability of the host to clear $P$. gingivalis depends on MyD88. Other studies have identified an important role for TRIF in the 


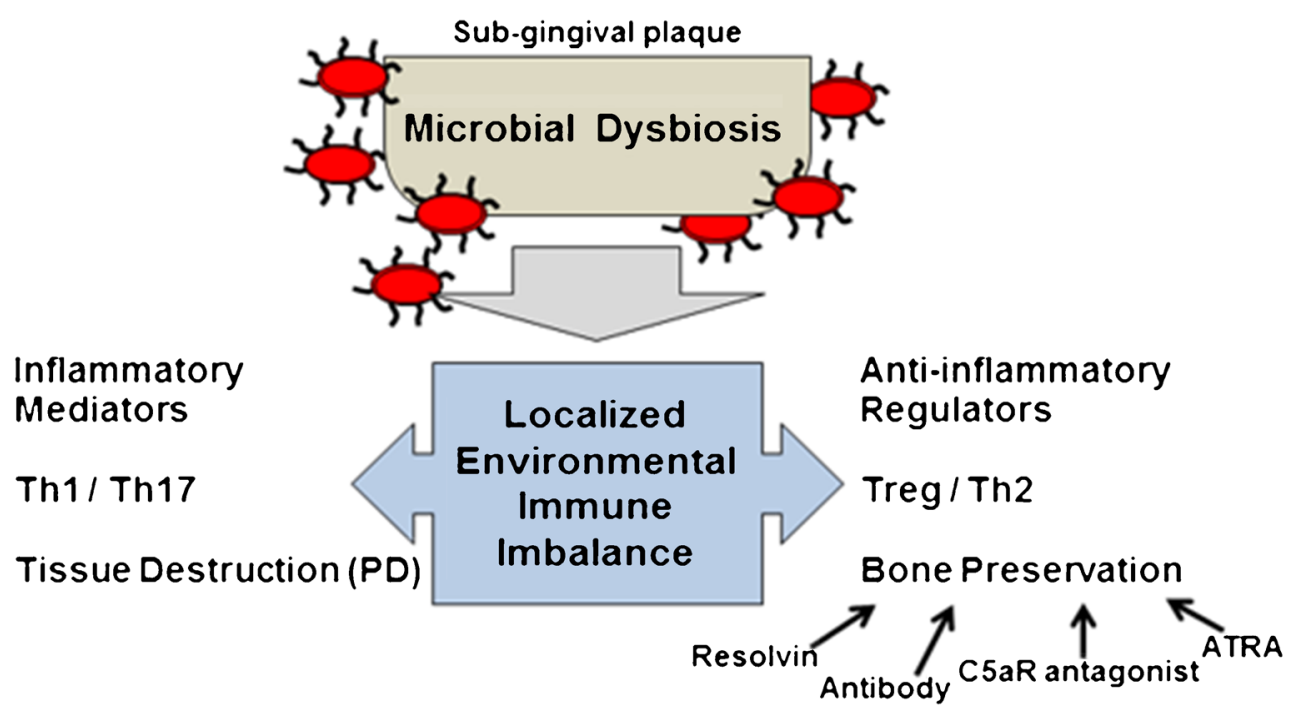

Fig. 1 Microbial dysbiosis and immune imbalance. In periodontal health the presence of defined periodontal pathogens is low. Emerging data support that rather than the presence of specific organisms, it may be that keystone pathogens such as $P$. gingivalis (red ovals) generate a microbial dysbiosis in the sub-gingival plaque. In response, at a micro-environmental level, immune imbalance ensues as a consequence of the immune response, pathogen effects, and infiltration of specific T cell subsets that shift the balance locally towards inflammation (characterized by pro-

response to $P$. gingivalis. TRIF participates in shaping CD4 T cell responses to $P$. gingivalis $\mathrm{HagB}$ [56], and using mouse MØ we recently observed a role for TRIF in full development of cellular TNF- $\alpha$ responses to P. gingivalis [57]. Thus, MyD88-dependent and MyD88-independent signaling both appear to play important roles in different phases of the host response during $\mathrm{PD}$.

TRIF, Interferon Regulatory Factors and Type 1 Interferon

CXCL10 (IP-10) and CCL5 (RANTES) are detected in PD [58]. The expression of these chemokines is dependent on interferon regulatory factor 3 (IRF3) [59]. In the context of TLR-signaling activation of IRF3 occurs via TLR3 and TLR4 through TRIF via TBK-1 [60]. The TLR / TRIF / IRF3 axis is essential in the production of type 1 interferon (IFN $\alpha$ and IFN $\beta$ ) [61]. IFN $\beta$ serves as an activator of additional gene products in an autocrine manner through type 1 interferon receptor (T1IFNr), STAT1/2 pathway to drive expression of interferon inducible genes such as IP-10 and RANTES [61]. Thus it is highly plausible that factors under the control of IRF3 play an important role in PD. Indeed, elevated IFN $\alpha$ have been measured in tissue samples from periodontitis patients $[18,62]$, and following periodontal therapy type 1 interferon levels dropped to those observed in healthy subjects [63]. Gaddis et al. [64] reported that recombinant $P$. gingivalis HagB stimulates bone marrow derived DCs to express IRF3 and IFN $\beta$. Recently we reported that IRF3 participates in the development of a full immune response to $P$. gingivalis [57]. inflammatory mediators and Th1 and Th17 cells), and eventually leading to tissue destruction (PD). Numerous factors are at play to counterbalance the destructive inflammation, such as anti-inflammatory and immuno-regulatory cytokine expression, Treg and Th2 cells. Although not fully defined, contributions of antibody, resolvin [35], C5aR antagonist [81], and all-trans retinoic acid (ATRA) treatments [42] may represent important therapeutic advances, by shifting the balance of the host response toward oral bone preservation

Furthermore, IRF7 participates in TLR2 and TLR7 signaling by MØ cultured with P. gingivalis or its LPS [65]. To date, the contribution of the TRIF-signaling pathway to the key clinically relevant endpoint of oral bone loss has not been reported. A recent paper supports that type 1 interferon may be important to the pathogenesis of PD. Nowak and colleagues identified an important mechanistic difference between local and generalized PD based on presence of type I NKT cells in aggressive, but not chronic, periodontitis lesions in vivo, and in vitro modeling using DCs identified that $A$. actinomycetemcomitans, but not $P$. gingivalis, elicited a type 1 IFN signature [66 ]. Collectively, these findings bring together important elements of MyD88 and TRIF signaling pathways as critical to the development of PD. The implications of the TRIF pathway in the progression of PD and oral bone homeostasis will require continued investigation.

Other Pattern Recognition Receptors and PD

\section{Scavenger Receptors}

Scavenger receptors are a family of membrane bound innate immune PRRs [67]. These receptors are best defined by their roles in lipid scavenging by MØ [67]; however, two of these receptors, scavenger receptor (SR)-A and CD36, participate in bone remodeling and the host response to periodontal pathogens. Lin et al. [68] reported that mice deficient in SR-A had greater levels of bone mineral density than WT mice. Our studies support that $P$. gingivalis stimulates SR-A $(m s r 1)$ gene 
and surface protein expression and that the presence of SR-A in part limits host TNF- $\alpha$ production to this organism [69]. Triantafilou et al. [70] showed that CD36 was present in the lipid rafts of vascular endothelial cells and was one of multiple receptors used by human vascular endothelial cells to bind $P$. gingivalis, including TLR1, TLR2, and CD11b/CD18. To date, no studies have been performed to broadly assess scavenger receptor expression in human PD lesions, define the contribution of scavenger receptors in PD bone remodeling, or follow-up the role these receptors may have in shaping subgingival microbial communities; and as such follow-up studies are necessary.

\section{Nucleotide-Binding Oligimerization Domain (NOD)-Like Receptors}

The nucleotide-binding oligomerization domain or (NOD)like receptors (NLRs) constitute a family of intracellular sensors of microbial PAMPs and host DAMPs that enter cells following uptake [71]. In one scheme, NLRs are divided into 3 groups: NODs (NOD1, NOD2, and others); NLRPs (also called NALPs); and IPAF (recently reviewed [72]). In the context of PD, NOD utilization has emerged as an important sensing mechanism. NOD1 and NOD2 are associated with ICAM-1 expression in human gingival fibroblasts responding to $\mathrm{P}$. gingivalis challenge [73]. In addition to $P$. gingivalis, $A$. actinomycetemcomitans, and $F$. nucleatum stimulate NODs, and interestingly, in comparison to the other organisms, $P$. gingivalis was a less potent activator [74]. In a mouse model of oral bone loss, mice lacking NOD1 exhibit reduced bone loss, impaired recruitment of neutrophils to gingival tissues, and reduced osteoclasts in alveolar bone [75]. NOD2 deficiency in hyperlipidemic ApoE gene knockout mice led to significant increases in inflammatory cytokine production and alveolar bone loss, compared with ApoE-knockout mice, in response to $P$. gingivalis oral challenge [76]. Interestingly NOD-mediated evasion by periodontal pathogens may serve as important mechanisms to their survival. Madrigal et al. [77] reported that $P$. gingivalis likely evades a NOD-mediated host response through the ability to degrade RIPK1 and RIPK2 via its lysine-specific gingipain, Kgp. When taken collectively these findings point to NODs as important mediators of pathogen-elicited inflammation and oral bone loss.

\section{Complement and Antibody}

\section{Complement}

Complement is an important molecular system that provides antimicrobial activity. Indeed, in the context of PD, complement components are found in crevicular fluids [78], and new findings support an important role for the complement system in the pathogenesis of PD. We will not go in depth into the role of complement here, as this has recently been expertly reviewed [79]. However, from a translational perspective the observation that $\mathrm{C} 5 \mathrm{a}$ receptor $(\mathrm{C} 5 \mathrm{aR})$-deficient mice are protected from $P$. gingivalis-elicited oral bone loss [80], and that treatment with a $\mathrm{C} 5 \mathrm{aR}$ antagonist protects mice from bone loss induced by oral $P$. gingivalis challenge, is very exciting [81].

\section{Antibody}

Evidence of adaptive immune activation in PD is generally established [82]; however, what these antibodies are doing in the context of disease is not clear. Approaches looking deeper into antibody function support their ability to opsonize, and in the context of $P$. gingivalis, treatment with $\mathrm{IgG}$ purified from patient sera support opsonophagocytic uptake by neutrophils [83]. Yet the presence of elevated levels of antibodies to bacteria associated with PD does not stop the progression of disease [84]. Therefore, the antibodies in PD may be generated to pathogen epitopes that are not highly expressed or are poorly accessible, or are unable to effectively interact with bacteria when they reside in biofilm. Indeed, a myriad of factors may be involved, and future studies in this area will impact on the ability to design an effective vaccine to the major periodontal pathogens.

\section{Macrophages as Effectors at the Microenvironmental Level}

Although neutrophils and lymphocytes are important cells in the pathogenesis of PD [21, 85], roles for other immune cells such as MØ are less well understood [86-88]. MØ comprise between $5 \%$ and $30 \%$ of the inflammatory cells identified in PD lesions [89]. These cells are recruited in response to inflammatory and chemotactic signaling, and upon entering diseased periodontal tissues express pro-inflammatory and anti-inflammatory mediators [20,25], and may influence a variety of other effector functions.

MØ are heterogeneous and highly dynamic cells that provide important homeostatic and immune functions [90]. Early studies identified MØ subsets employing CD14 and CD16 [90]; however, recent studies now clearly depict a highly dynamic and flexible plasticity of MØ to adjust to the microenvironmental needs on entering local tissues [91]. The term "polarization" has been used generically to describe this functional adaptation; however, this terminology is restrictive as it suggests antitheses of what is thought a dynamic continuum of MØ activation types [92]. Early studies showed that classical $\mathrm{M} \varnothing$ activation in response to pathogen is replicated by IFN- $\gamma$ + TLR agonist (LPS) treatment. Gordon and colleagues showed that MØ were also activated by IL-4 treatment; 
however, this activation was different than classical activation, as in addition to other molecules, nitric oxide (NO) production was not detected - and was coined alternative activation [93]. Three groups of alternatively activated $\mathrm{M \varnothing}$ are frequently studied and include M2a, M2b, and M2c, based on differences in MØ response to IL-4, immune complexes, and IL-10, respectively [94, 95]. Broadly, alternatively activated MØ are associated with resolution of inflammation, tissue repair, and interestingly in chronic infections such as brucellosis, plague, and Q fever [96].

In the study of PD and other diseases, $\mathrm{MØ}$ are often used as model cells to define immune function. Indeed, transfer of TLR2 expressing MØ to TLR2-deficient mice restored host sensitivity to $P$. gingivalis oral challenge [97]. However, at the subset level, the contribution of these cells to PD is poorly understood. An elevated level of IFN- $\gamma$, a prototypical stimulus for classical MØ activation is detected in PD samples providing an environment potentially supporting classically activated MØ (25). Employing immunohistochemical approaches Chapple et al. [98] detected acute inflammatory

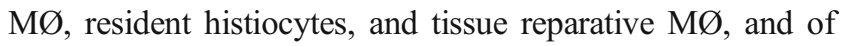
these, the most prevalent were reparative MØ. Topoll et al. [99] reported significant shifts in anti-inflammatory MØ population. In the context of prototypical stimulus for alternative activation, IL-4 levels appear low during active PD, and if provided at high levels, IL-4 can drive MØ apoptosis [100]. An inverse relationship between IL-4 levels and periodontal status has been observed with elevated IL-4 associated with remission/improvement of periodontal status [101]. The potential for IL-10 to influence alternative activation of MØ in PD is not well understood. Taken together, these data support that MØ phenotypic variation may be associated with different stages of PD based on micro-environmental levels of inflammatory and regulatory mediators and bacterial composition of sub-gingival plaque. Future studies directed to better understand the impact MØ have on PD inflammation, tissue repair, and oral bone homeostasis may provide important insights into the dynamics of the underlying mechanisms.

\section{Novel Therapeutic Approaches}

Although a vaccine for PD is attractive, to date, none exists. Filling this void is an array of novel and exciting therapeutic approaches aimed at interceding in the progression of PD by targeting the host immune response. In a rabbit model, application of resolvin R1 prevented periodontitis [35]. In a mouse model, C5aR antagonist significantly limited $P$. gingivaliselicited oral bone loss [81]. Furthermore, treatment of mice with all-trans retinoic acid (ATRA), as an activator of Treg cells, reduced levels of alveolar bone loss by modulating the Th17/Treg imbalance to Treg predominance [42••]. Clinical studies are needed to determine the translational capacity of these treatment approaches to limit human disease.

\section{Conclusions}

Despite the advances made in the field of PD pathogenesis and the roles played by innate and adaptive immunity, much more needs to be understood. The implication that periodontal pathogens cause an immune imbalance that leads to a general microbial dysbiosis is an important new concept backed by compelling findings. The implications this new information may have at the micro-environmental level may provide a new window to better predict where oral bone homeostasis may be disrupted. Indeed, with advances in potentially relevant biomarkers, better targeting of disease therapy based on novel pathogen composition and immunologic understanding, in the near future better control of PD progression may be on the horizon.

Acknowledgements These studies were supported by PHS grants DE021497 and AI078894 to FCG.

\section{Compliance with Ethics Guidelines}

Conflict of Interest Dr. Nasi Huang and Dr. Frank C. Gibson received a grant from $\mathrm{NIH}$ for ongoing research projects.

Human and Animal Rights and Informed Consent This article does not contain any studies with human or animal subjects performed by any of the authors.

\section{References}

Papers of particular interest, published recently, have been highlighted as:

- Of importance

•. Of major importance

1. Benakanakere M, Kinane DF. Innate cellular responses to the periodontal biofilm. Front Oral Biol. 2012;15:41-55.

2. Socransky SS, Haffajee AD, Cugini MA, Smith C, Kent Jr RL. Microbial complexes in subgingival plaque. J Clin Periodontol. 1998;25:134-44.

3. Holt SC, Ebersole J, Felton J, Brunsvold M, Kornman KS. Implantation of Bacteroides gingivalis in nonhuman primates initiates progression of periodontitis. Science. 1988;239:55-7.

4. Kesavalu L, Sathishkumar S, Bakthavatchalu V, et al. Rat model of polymicrobial infection, immunity, and alveolar bone resorption in periodontal disease. Infect Immun. 2007;75:1704-12.

5. Polak D, Wilensky A, Shapira L, et al. Mouse model of experimental periodontitis induced by Porphyromonas gingivalis/ Fusobacterium nucleatum infection: bone loss and host response. J Clin Periodontol. 2009;36:406-10.

6. Paster BJ, Boches SK, Galvin JL, et al. Bacterial diversity in human subgingival plaque. J Bacteriol. 2001;183:3770-83. 
7. Dewhirst FE, Chen T, Izard J, et al. The human oral microbiome. J Bacteriol. 2010;192:5002-17.

8. Colombo AP, Bennet S, Cotton SL, et al. Impact of periodontal therapy on the subgingival microbiota of severe periodontitis: comparison between good responders and individuals with refractory periodontitis using the human oral microbe identification microarray. J Periodontol. 2012;83:1279-87. Use of the human oral microbiome microarray invesitgators identified novel organisms and groups of organisms associated with refractory PD.

9. Darveau RP. Periodontitis: a polymicrobial disruption of host homeostasis. Nat Rev Microbiol. 2010;8:481-90.

10. Hajishengallis G, Liang S, Payne MA, et al. Low-abundance biofilm species orchestrates inflammatory periodontal disease through the commensal microbiota and complement. Cell Host Microbe. 2011;10:497-506.

11. Darveau RP, Hajishengallis G, Curtis MA. Porphyromonas gingivalis as a potential community activist for disease. J Dent Res. 2012;91:816-20.

12. Weinberg A, Belton CM, Park Y, Lamont RJ. Role of fimbriae in Porphyromonas gingivalis invasion of gingival epithelial cells. Infect Immun. 1997;65:313-6.

13. Coats SR, Berezow AB, To TT, et al. The lipid A phosphate position determines differential host Toll-like receptor 4 responses to phylogenetically related symbiotic and pathogenic bacteria. Infect Immun. 2011;79:203-10.

14. Nichols FC, Housley WJ, O'Conor CA, Manning T, Wu S, Clark $\mathrm{RB}$. Unique lipids from a common human bacterium represent a new class of Toll-like receptor 2 ligands capable of enhancing autoimmunity. Am J Pathol. 2009;175:2430-8.

15. Guo Y, Nguyen KA, Potempa J. Dichotomy of gingipains action as virulence factors: from cleaving substrates with the precision of a surgeon's knife to a meat chopper-like brutal degradation of proteins. Periodontol 2000. 2010;54:15-44.

16. Sheets SM, Robles-Price AG, McKenzie RM, Casiano CA, Fletcher HM. Gingipain-dependent interactions with the host are important for survival of Porphyromonas gingivalis. Front Biosci. 2008; 13:3215-38.

17. Blach A, Franek E, Witula A, et al. The influence of chronic periodontitis on serum TNF-alpha, IL-6 and hs-CRP concentrations, and function of graft and survival of kidney transplant recipients. Clin Transplant. 2009;23:213-9.

18. Kajita K, Honda T, Amanuma R, et al. Quantitative messenger RNA expression of Toll-like receptors and interferon-alphal in gingivitis and periodontitis. Oral Microbiol Immunol. 2007;22: 398-402.

19. Kinane DF, Mooney J, Ebersole JL. Humoral immune response to Actinobacillus actinomycetemcomitans and Porphyromonas gingivalis in periodontal disease. Periodontol 2000. 1999;20: 289-340.

20. Gamonal J, Acevedo A, Bascones A, Jorge O, Silva A. Levels of interleukin- 1 beta, -8 , and -10 and RANTES in gingival crevicular fluid and cell populations in adult periodontitis patients and the effect of periodontal treatment. J Periodontol. 2000;71:1535-45.

21. Cekici A, Kantarci A, Hasturk H, Van Dyke TE. Inflammatory and immune pathways in the pathogenesis of periodontal disease. Periodontol 2000. 2014;64:57-80.

22. Cochran DL. Inflammation and bone loss in periodontal disease. J Periodontol. 2008;79:1569-76.

23. Yuan H, Gupte R, Zelkha S, Amar S. Receptor activator of nuclear factor kappa B ligand antagonists inhibit tissue inflammation and bone loss in experimental periodontitis. J Clin Periodontol. 2011;38:1029-36.

24. Tonetti MS, Imboden MA, Gerber L, Lang NP, Laissue J, Mueller C. Localized expression of mRNA for phagocyte-specific chemotactic cytokines in human periodontal infections. Infect Immun. 1994;62:4005-14.
25. Salvi GE, Brown CE, Fujihashi K, et al. Inflammatory mediators of the terminal dentition in adult and early onset periodontitis. J Periodontal Res. 1998;33:212-25.

26. Taubman MA, Kawai T. Involvement of T-lymphocytes in periodontal disease and in direct and indirect induction of bone resorption. Crit Rev Oral Biol Med. 2001;12:125-35.

27. Mosmann TR, Cherwinski H, Bond MW, Giedlin MA, Coffman RL. Two types of murine helper T cell clone. I. Definition according to profiles of lymphokine activities and secreted proteins. $\mathrm{J}$ Immunol. 1986;136:2348-57.

28. Gemmell E, Seymour GJ. Immunoregulatory control of Th1/Th2 cytokine profiles in periodontal disease. Periodontol 2000. 2004;35:21-41.

29. Ebersole JL, Taubman MA. The protective nature of host responses in periodontal diseases. Periodontol 2000. 1994;5:11241.

30. Bannenberg G, Serhan CN. Specialized pro-resolving lipid mediators in the inflammatory response: an update. Biochim Biophys Acta. 1801;2010:1260-73.

31. Freire MO, Van Dyke TE. Natural resolution of inflammation. Periodontol. 2000;201363:149-64.

32. Yang R, Chiang N, Oh SF, Serhan CN. Metabolomics-lipidomics of eicosanoids and docosanoids generated by phagocytes. Curr Protoc Immunol. 2011; Chapter 14:Unit 1426.

33. Hasturk H, Kantarci A, Goguet-Surmenian E, et al. Resolvin E1 regulates inflammation at the cellular and tissue level and restores tissue homeostasis in vivo. J Immunol. 2007;179:7021-9.

34. Schwab JM, Chiang N, Arita M, Serhan CN. Resolvin E1 and protectin D1 activate inflammation-resolution programmes. Nature. 2007;447:869-74.

35. Hasturk H, Kantarci A, Ohira T, et al. RvE1 protects from local inflammation and osteoclast- mediated bone destruction in periodontitis. FASEB J. 2006;20:401-3.

36. Nakajima T, Ueki-Maruyama K, Oda T, et al. Regulatory T-cells infiltrate periodontal disease tissues. J Dent Res. 2005;84:639-43.

37. Aranha AM, Repeke CE, Garlet TP, et al. Evidence supporting a protective role for th9 and th 22 cytokines in human and experimental periapical lesions. J Endod. 2013;39:83-7.

38. Akdis M, Palomares O, van de Veen W, van Splunter M, Akdis CA. TH17 and TH22 cells: a confusion of antimicrobial response with tissue inflammation versus protection. J Allergy Clin Immunol. 2012;129:1438-49. quiz1450-1431.

39. Cardoso CR, Garlet GP, Crippa GE, et al. Evidence of the presence of T helper type 17 cells in chronic lesions of human periodontal disease. Oral Microbiol Immunol. 2009;24:1-6.

40. Garlet GP, Cardoso CR, Mariano FS, et al. Regulatory T cells attenuate experimental periodontitis progression in mice. $\mathrm{J}$ Clin Periodontol. 2010;37:591-600.

41.• Eskan MA, Jotwani R, Abe T, et al. The leukocyte integrin antagonist Del-1 inhibits IL-17-mediated inflammatory bone loss. Nat Immunol. 2012;13:465-73. Linkage of IL-17 to infectionelicited oral bone loss phenotype and defined the role of Del-1 an endogenous inhibitor of neutrophil adherence.

42.• Wang L, Wang J, Jin Y, Gao H, Lin X. Oral administration of alltrans retinoic acid suppresses experimental periodontitis by modulating the Th17/Treg imbalance. J Periodontol. 2013. Treatment of mice with all-trans retanoic acid during $P$. gingivalis infection shifts balance to Treg predominance, reducing cellular infiltration into gingival tissues, and preserved oral bone.

43. Adibrad M, Deyhimi P, Ganjalikhani Hakemi M, Behfarnia P, Shahabuei M, Rafiee L. Signs of the presence of Th17 cells in chronic periodontal disease. J Periodontal Res. 2012;47:525-31.

44. Martin M, Katz J, Vogel SN, Michalek SM. Differential induction of endotoxin tolerance by lipopolysaccharides derived from Porphyromonas gingivalis and Escherichia coli. J Immunol. 2001;167:5278-85. 
45. Cohen N, Morisset J, Emilie D. Induction of tolerance by Porphyromonas gingivalis on APCS: a mechanism implicated in periodontal infection. J Dent Res. 2004;83:429-33.

46. Jotwani R, Palucka AK, Al-Quotub M, et al. Mature dendritic cells infiltrate the T cell-rich region of oral mucosa in chronic periodontitis: in situ, in vivo, and in vitro studies. J Immunol. 2001;167:4693-700.

47. Huang CB, Altimova Y, Strange S, Ebersole JL. Polybacterial challenge effects on cytokine/chemokine production by macrophages and dendritic cells. Inflamm Res. 2011;60:119-25.

48. Herath TD, Darveau RP, Seneviratne CJ, Wang CY, Wang Y, Jin L. Tetra- and penta-acylated lipid A structures of Porphyromonas gingivalis LPS differentially activate TLR4-mediated NF-kappaB signal transduction cascade and immuno-inflammatory response in human gingival fibroblasts. PLoS One. 2013;8:e58496.

49. Nussbaum G, Ben-Adi S, Genzler T, Sela M, Rosen G. Involvement of Toll-like receptors 2 and 4 in the innate immune response to Treponema denticola and its outer sheath components. Infect Immun. 2009;77:3939-47.

50. Burns E, Bachrach G, Shapira L, Nussbaum G. Cutting Edge: TLR2 is required for the innate response to Porphyromonas gingivalis: activation leads to bacterial persistence and TLR2 deficiency attenuates induced alveolar bone resorption. J Immunol. 2006;177:8296-300.

51. Gibson 3rd FC, Genco CA. Porphyromonas gingivalis mediated periodontal disease and atherosclerosis: disparate diseases with commonalities in pathogenesis through TLRs. Curr Pharm Des. 2007;13:3665-75.

52. Costalonga M, Batas L, Reich BJ. Effects of Toll-like receptor 4 on Porphyromonas gingivalis-induced bone loss in mice. J Periodontal Res. 2009;44:537-42.

53. Kumar H, Kawai T, Akira S. Toll-like receptors and innate immunity. Biochem Biophys Res Commun. 2009;388:621-5.

54. Hoebe K, Du X, Georgel P, et al. Identification of Lps2 as a key transducer of MyD88-independent TIR signalling. Nature. 2003;424:743-8.

55. Burns E, Eliyahu T, Uematsu S, Akira S, Nussbaum G. TLR2dependent inflammatory response to Porphyromonas gingivalis is MyD88 independent, whereas MyD88 is required to clear infection. J Immunol. 2010;184:1455-62.

56. Gaddis DE, Michalek SM, Katz J. TLR4 signaling via MyD88 and TRIF differentially shape the CD4 $+\mathrm{T}$ cell response to Porphyromonas gingivalis hemagglutinin B. J Immunol. 2011;186: 5772-83.

57. Shaik-Dasthagirisaheb YB, Huang N, Gibson FC, 3rd. Inflammatory response to Porphyromonas gingivalis partially requires interferon regulatory factor (IRF) 3. Innate Immun. 2014;20:312-9.

58. Kabashima H, Yoneda M, Nagata K, Hirofuji T, Maeda K. The presence of chemokine (MCP-1, MIP-1alpha, MIP-1beta, IP-10, RANTES)-positive cells and chemokine receptor (CCR5, CXCR3)-positive cells in inflamed human gingival tissues. Cytokine. 2002;20:70-7.

59. McWhirter SM, Fitzgerald KA, Rosains J, Rowe DC, Golenbock DT, Maniatis T. IFN-regulatory factor 3-dependent gene expression is defective in Tbk1-deficient mouse embryonic fibroblasts. Proc Natl Acad Sci U S A. 2004;101:233-8.

60. Fitzgerald KA, Rowe DC, Barnes BJ, et al. LPS-TLR4 signaling to IRF-3/7 and NF-kappaB involves the toll adapters TRAM and TRIF. J Exp Med. 2003;198:1043-55.

61. Decker T, Muller M, Stockinger S. The Yin and Yang of type I interferon activity in bacterial infection. Nat Rev Immunol. 2005;5:675-87.

62. Prabhu A, Michalowicz BS, Mathur A. Detection of local and systemic cytokines in adult periodontitis. J Periodontol. 1996;67:515-22.

63. Wright HJ, Matthews JB, Chapple IL, Ling-Mountford N, Cooper PR. Periodontitis associates with a type 1 IFN signature in peripheral blood neutrophils. J Immunol. 2008;181:5775-84.
64. Gaddis DE, Michalek SM, Katz J. Requirement of TLR4 and CD14 in dendritic cell activation by Hemagglutinin B from Porphyromonas gingivalis. Mol Immunol. 2009;46:2493-504.

65. Zhou Q, Amar S. Identification of signaling pathways in macrophage exposed to Porphyromonas gingivalis or to its purified cell wall components. J Immunol. 2007;179:7777-90.

66. Nowak M, Kramer B, Haupt M, et al. Activation of invariant NK T cells in periodontitis lesions. J Immunol. 2013;190:2282-91. Specific differences in type 1 NKT cell infiltration into periodontal lesions, as well as ability of cells to differentially respond by producing type 1 IFN may be important difference to the mechanistic progression of aggressive and chronic forms of $P D$.

67. Platt N, Haworth R, Darley L, Gordon S. The many roles of the class A macrophage scavenger receptor. Int Rev Cytol. 2002;212: $1-40$.

68. Lin YL, de Villiers WJ, Garvy B, et al. The effect of class A scavenger receptor deficiency in bone. J Biol Chem. 2007;282: 4653-60.

69. Baer MT, Huang N, Gibson 3rd FC. Scavenger receptor A is expressed by macrophages in response to Porphyromonas gingivalis, and participates in TNF-alpha expression. Oral Microbiol Immunol. 2009;24:456-63.

70. Triantafilou M, Gamper FG, Lepper PM, et al. Lipopolysaccharides from atherosclerosis-associated bacteria antagonize TLR4, induce formation of TLR2/1/CD36 complexes in lipid rafts and trigger TLR2-induced inflammatory responses in human vascular endothelial cells. Cell Microbiol. 2007;9:2030-9.

71. Elinav E, Strowig T, Henao-Mejia J, Flavell RA. Regulation of the antimicrobial response by NLR proteins. Immunity. 2011;34:665-79.

72. Wen H, Miao EA, Ting JP. Mechanisms of NOD-like receptorassociated inflammasome activation. Immunity. 2013;39:432-41.

73. Liu J, Duan J, Wang Y, Ouyang X. Intracellular adhesion molecule-1 is regulated by Porphyromonas gingivalis through nucleotide binding oligomerization domain-containing proteins 1 and 2 molecules in periodontal fibroblasts. J Periodontol. 2014;85:358-68.

74. Okugawa T, Kaneko T, Yoshimura A, Silverman N, Hara Y. NOD1 and NOD2 mediate sensing of periodontal pathogens. J Dent Res. 2010;89:186-91.

75. Jiao Y, Darzi Y, Tawaratsumida K, et al. Induction of bone loss by pathobiont-mediated Nod1 signaling in the oral cavity. Cell Host Microbe. 2013;13:595-601.

76. Yuan H, Zelka S, Burkatovskaya M, Gupte R, Leeman SE, Amar S. Pivotal role of NOD2 in inflammatory processes affecting atherosclerosis and periodontal bone loss. Proc Natl Acad Sci U S A. 2013;110:E5059-68.

77. Madrigal AG, Barth K, Papadopoulos G, Genco CA. Pathogenmediated proteolysis of the cell death regulator RIPK1 and the host defense modulator RIPK2 in human aortic endothelial cells. PLoS Pathog. 2012;8:e1002723.

78. Attstrom R, Laurel AB, Lahsson U, Sjoholm A. Complement factors in gingival crevice material from healthy and inflamed gingiva in humans. J Periodontal Res. 1975;10:19-27.

79. Hajishengallis G, Abe T, Maekawa T, Hajishengallis E, Lambris JD. Role of complement in host-microbe homeostasis of the periodontium. Semin Immunol. 2013;25:65-72.

80. Liang S, Krauss JL, Domon H, et al. The C5a receptor impairs IL12-dependent clearance of Porphyromonas gingivalis and is required for induction of periodontal bone loss. J Immunol. 2011;186:869-77.

81. Abe T, Hosur KB, Hajishengallis E, et al. Local complementtargeted intervention in periodontitis: proof-of-concept using a C5a receptor (CD88) antagonist. J Immunol. 2012;189:5442-8.

82. Haffajee AD, Socransky SS, Taubman MA, Sioson J, Smith DJ. Patterns of antibody response in subjects with periodontitis. Oral Microbiol Immunol. 1995;10:129-37. 
83. Gibson FC, Savelli J, Van Dyke TE, Genco CA. Gingipainspecific IgG in the sera of patients with periodontal disease is necessary for opsonophagocytosis of Porphyromonas gingivalis. J Periodontol. 2005;76:1629-36.

84. Whitney C, Ant J, Moncla B, Johnson B, Page RC, Engel D. Serum immunoglobulin $\mathrm{G}$ antibody to Porphyromonas gingivalis in rapidly progressive periodontitis: titer, avidity, and subclass distribution. Infect Immun. 1992;60:2194-200.

85. Pihlstrom BL, Michalowicz BS, Johnson NW. Periodontal diseases. Lancet. 2005;366:1809-20.

86. Charon J, Toto PD, Gargiulo AW. Activated macrophages in human periodontitis. J Periodontol. 1981;52:328-35.

87. Hasturk H, Kantarci A, Van Dyke TE. Oral inflammatory diseases and systemic inflammation: role of the macrophage. Front Immunol. 2012;3:118.

88. Sima C, Glogauer M. Macrophage subsets and osteoimmunology: tuning of the immunological recognition and effector systems that maintain alveolar bone. Periodontol 2000. 2013;63:80-101.

89. Okada H, Murakami S. Cytokine expression in periodontal health and disease. Crit Rev Oral Biol Med. 1998;9:248-66.

90. Gordon S, Taylor PR. Monocyte and macrophage heterogeneity. Nat Rev Immunol. 2005;5:953-64.

91. Mosser DM. The many faces of macrophage activation. J Leukoc Biol. 2003;73:209-12.

92. Mosser DM, Edwards JP. Exploring the full spectrum of macrophage activation. Nat Rev Immunol. 2008;8:958-69.
93. Martinez FO, Helming L, Gordon S. Alternative activation of macrophages: an immunologic functional perspective. Annu Rev Immunol. 2009;27:451-83.

94. Mantovani A, Sica A, Sozzani S, Allavena P, Vecchi A, Locati M. The chemokine system in diverse forms of macrophage activation and polarization. Trends Immunol. 2004;25:677-86.

95. Martinez FO, Sica A, Mantovani A, Locati M. Macrophage activation and polarization. Front Biosci. 2008;13:453-61.

96. Benoit M, Desnues B, Mege JL. Macrophage polarization in bacterial infections. J Immunol. 2008;181:3733-9.

97. Papadopoulos G, Weinberg EO, Massari P, et al. Macrophagespecific TLR2 signaling mediates pathogen-induced TNFdependent inflammatory oral bone loss. J Immunol. 2013;190: 1148-57.

98. Chapple CC, Srivastava M, Hunter N. Failure of macrophage activation in destructive periodontal disease. J Pathol. 1998;186:281-6.

99. Topoll HH, Zwadlo G, Lange DE, Sorg C. Phenotypic dynamics of macrophage subpopulations during human experimental gingivitis. J Periodontal Res. 1989;24:106-12.

100. Yamamoto M, Kawabata K, Fujihashi K, et al. Absence of exogenous interleukin-4-induced apoptosis of gingival macrophages may contribute to chronic inflammation in periodontal diseases. Am J Pathol. 1996;148:331-9.

101. Pradeep AR, Roopa Y, Swati PP. Interleukin-4, a T-helper 2 cell cytokine, is associated with the remission of periodontal disease. $\mathrm{J}$ Periodontal Res. 2008;43:712-6. 